\title{
On the Gamma-Ray Bursts Origin
}

\author{
A. G. Syromyatnikov \\ Department of Physics, St. Petersburg State University, St. Petersburg, Russia \\ Email:alsyromyatnikov@mail.ru
}

How to cite this paper: Syromyatnikov, A.G. (2016) On the Gamma-Ray Bursts Origin. International Journal of Astronomy and Astrophysics, 6, 425-434. http://dx.doi.org/10.4236/ijaa.2016.64034

Received: October 27, 2016

Accepted: December 18, 2016

Published: December 21, 2016

Copyright $\odot 2016$ by author and Scientific Research Publishing Inc. This work is licensed under the Creative Commons Attribution International License (CC BY 4.0).

http://creativecommons.org/licenses/by/4.0/

\begin{abstract}
Gamma-ray (GRBs) and X-ray Bursts are millisecond-10 and 1000 seconds-long events of unknown origin. Recent simulations of the merger of binary neutron star systems do not generate a magnetically dominated called funnel nor a relativistic outflow. New models for the detection the afterglow of GRB 121024A, measured 0.15 days after the burst, invoke anisotropy as required to produce the complex microphysics of realistic shocks in relativistic jets. On the other hand the non-thermal gamma-rays are supposed to be produced by a fireball of relativistic $\mathrm{e}^{-} \mathrm{e}^{+}$pairs that are created by annihilation of neutrino-antineutrino pairs in the vicinity of the hot, merged object. It is also known that in a system of a large number of fermions with pairs, gravitational interaction occurs a spontaneous breaking of the vacuum spatial symmetry, accompanied by gravitational mass defect. If spherical symmetry is broken, as in the known case of the merger scenario where a rapidly rotating disk can be formed and material is pulled away from rotation axis by centrifugal forces, then a baryon-free funnel along the rotation axes may allow relativistic beam of $\gamma^{\prime} \mathrm{s}$ and $\mathrm{e}^{-} \mathrm{e}^{+}$ to escape. It might lead to matter ejection with Lorentz factors of $\sim 10^{2}-10^{3}$ which are in the right range to enable copious gamma production during shock interaction with ambient interstellar gas. Here we show that the space rays generation mechanism on a method of direct transformation of intergalactic gamma-rays to the proton current on spin shock-waves ensure precise agreement between generated proton currents (spin shock waves theory) with the angular distribution data of Galactic gamma-rays as well as for the individual pulses of gamma-/X-ray bursts. There is a precise confirmation of the generated currents (theory) with the burst radiation data characterized by the standard deviation of $\pm 1 \%$ in intensity in relative units within the sensitivity of the equipment. Thus, it was found that the spin angular momentum conservation law (equation of dynamics of spin shock waves) in the X-ray/gamma ranges is fulfilled exactly in real time. The next step involves setting the inverse problem of determining the wave function disturbance on the differential of measured smoothing pulses. In the asymptotic large times the problem is reduced to the solutions of the functional equation with shift of the argument. This will give additional information about the change speed of the wave, as well as on the interaction.
\end{abstract}




\section{Keywords}

Gamma-Ray Bursts, Spin Density Waves, Spin Shock Waves, Gravitational Mass

Defect

\section{Introduction}

Gamma-ray (GRBs) and X-ray Bursts are millisecond-10 and 1000 seconds-long events of unknown origin. The nature of the jets and the role of magnetic fields in GRBs remain unclear. In article [1] about fully general-relativistic magnetohydrodynamic simulations of the merger of binary neutron star (BNS), systems were held a detailed investigation of the matter dynamics and of the magnetic field evolution, with particular attention to its global structure and possible emission of relativistic jets. However, in [1] neither a magnetically dominated called funnel nor a relativistic outflow are observed. New models for the detection the afterglow of GRB 121024A, measured 0.15 days after the burst, invoke anisotropy as required to produce the complex microphysics of realistic shocks in relativistic jets [2].

GRBs of low-energy gamma-ray are associated with core-collapse supernovae (SN) [3]. A theoretical understanding has emerged in the form of the fireball internal-external shocks model [3]. According to this model, GRBs are produced when the kinetic energy of an ultrarelativistic flow is dissipated in internal collisions. According to [4], measurements of the X-ray afterglow of very intense gamma-ray bursts (allow a critical test of GRB theories) disagree with that predicted by widely accepted fireball models of GRBs. In another side, some pioneer work on the Non-Occurrence of Type I X-ray Bursts from the Black Hole candidates is due to Mitra [5] and in this paper he concluded that "the Black Hole Candidates (BHCs) being uncharged and having finite masses cannot be Black Hole, because uncharged BHs have a unique mass $M=0$ ". "Thus the previous results that so-called BHCs are actually extremely hot, ultramagnetized, Magnetospheric Eternally Collapsing Objects rather than anything else get reconfirmation by nonoccurrence of Type I X-ray bursts in BHCs". On the same ground Tewari and Charan [6] [7] presented some mathematical evidences for Eternally Collapsing radiating Star and constructed Supernova models in certain conditions in which BHCs can form with some remnant. Initially both the mass and physical radius of pre Supernova remains 2.4 $M_{\odot}$ and $4.86 \mathrm{~km}$ respectively at the time of black hole formation [6]. You can see that this time does not fit a range of millisecond GBRs pulses.

The main objective of this work is to make sure the extensive materials of reviews on gamma-bursts at least within the range of millisecond pulses, and the nature of gamma-bursts is largely determined by the influence of powerful external sources. So when their exploration occurs, the space rays generation mechanism on a method of direct transformation of intergalactic gamma-rays to the proton current on spin shock-waves must be included in the consideration, which ensures precise agreement between generated proton currents (spin shock waves theory) with the angular distribution data of 
Galactic gamma-rays as well as for the individual pulses of X-ray bursts.

\section{Experimental Overview}

Also cusps of superconducting strings can serve as GRB engines [8]. A powerful beamed pulse of electromagnetic radiation from a cusp produced a jet of accelerated particles, whose propagation is terminated by the shock responsible for GRB. The string scale of symmetry breaking near $100 \mathrm{TeV}$ to which is close to the LHC, together with reasonable assumptions about the magnitude of cosmic magnetic fields and the fraction of volume that they occupy, explains the GRB rate, duration, and fluency, as well as the observed ranges of these quantities. This model predicts that GRBs are accompanied by strong bursts of gravitational radiation which should be detectable by LIGO, VIRGO, and LISA detectors. Another prediction is the diffuse $\mathrm{X}$ - and gamma-ray radiation at 8 MeV-100 GeV with a spectrum and flux comparable to the observed. The weakness of the model is the prediction of too low a rate of GRBs from galaxies, as compared with observations [8]. This suggests that [8] GRBs from cusps are responsible for only a subset of the observed GRBs not associated with galaxies.

Thus, anyway the success of numerous predictions requires consideration of internal-external sources. And yet must be (X-ray) afterglows.

On the other hand the non-thermal gamma-rays are supposed to be produced by a fireball of relativistic $\mathrm{e}^{-} \mathrm{e}^{+}$pairs that are created by annihilation of neutrino-antineutrino pairs in the vicinity of the hot, merged object [9]. It is also known [9] that a large number of fermions with a pair gravitational interaction occur a spatial symmetry spontaneous breaking, accompanied by gravitational mass defect (as all know the gravitational mass defect neutron stars reached $90 \%$ ). If spherical symmetry is broken, as in the known case of the merger scenario where a rapidly rotating dick can be formed and material is pulled away from rotation axis by centrifugal forces, then a baryon-free funnel along the rotation axes may allow relativistic beams of $\gamma s$ and $\mathrm{e}^{-} \mathrm{e}^{+}$to escape. It seems to be extremely hard to account for the energy of cosmological gamma-bursts by $v \bar{v}$ annihilations [9].

\section{The Range of Second Pulses}

According to the Conformal Gauge Theory of Gravitation (CGTG) [10] [11] [12], the BHs metric of the constant curvature is conformal-flat [13]. According to the CGTG in the Riemann-Cartan space of spherical symmetry it is indicates that as a result of the conformal conversion from the BHs metric to the Minkowski space flat metric the energy momentum tensor and the density are equal to zero. It like all other vacuum solution, as we see, is the solution of GR equations.

\section{Methods}

Another thing if it happens under the influence of gamma rays from a powerful external source. It might lead to matter ejection with Lorentz factors of $\sim 10^{2}-10^{3}$ which is in the right range to enable copious gamma production during shock interaction with 
ambient interstellar gas (see [9]). In another side there is an electromagnetic spin shock waves production from some gamma-rays sources [14]. Spin shock-waves, theoretically grounded on basic characteristics of the spin angular momentum density, as a part of a full angular momentum to spin shock-waves of electromagnetic nature in cold nonneutral plasma. Moreover it is taking into account the contribution of the famous two bubbles $20^{\circ}$ field in the angular distribution of gamma-radiation in addition to diffuse from the Galactic plane allowed to successfully conclude a precise agreement between generated proton currents (spin shock-waves theory) with the Galaxy gamma-radiation, characterized by the standard deviation of $\pm 1 \%$ in intensity in relative units within the sensitivity of the equipment [14]. This is done within frame of the space rays generation mechanism of a method of direct transformation of intergalactic gamma-rays to the proton current on spin shock-waves [14] [15] [16]. It should be mentioned that the "Eight spin shock-waves model" (ESSM), which is rounded based on an accurate solution of the Maxwell equations for a dust-like medium of charged particles (and which are identical to the spin conservation law) ion take into account those most required above spin polarization properties of previously unexplored. There are many quantum processes, all of which follow the spin conservation law of electromagnetic field. The Task was the following statement: Generating currents in the ground experiment of focusing radio waves to dielectric target (see link in [16]) is in the range of angles from $32^{\circ}$ to $104^{\circ}$ from the axis (the axis of anisotropy) in the direction towards incident radiation. The currents passed through the annular surface along the axis (the axis of anisotropy) source of the incident radiation flux. Reverse currents, extending in the direction of incident radiation, are limited to the limit angle $104^{\circ}$. Theory allows determining the distribution of currents in the corners, measured from the axis of anisotropy. Generate currents in the ESSM (see, e.g. [16]) in the inner configuration space is in the same range of angles from $32^{\circ}$ to $104^{\circ}$ from the axis of anisotropy. Observation of the Galactic radiation has the ability to measure its intensity only per unit area, rather than across the entire annular surface. However, based on the symmetry of the distribution of currents along the annular surface that takes place in a land lab experiment, in [14] [15] [16] it was performed and for the Galactic radiation. Then the comparison between the theory value of currents through the annular surface based on the Galactic longitude and intensity of gamma-radiation per unit of that surface becomes more defined.

\section{Equations}

The Eight spin shock waves model is grounded on basic of an accurate solution of the Maxwell equations for a dust-like medium of charged particles [17]. Actually Maxwell equations for a dust-like medium of charge particles in the flat space

$\partial^{\alpha} \partial_{[\alpha} A_{\mu]}=4 \pi / c \pi \rho_{e} u_{\mu}, u_{\mu}=e / c A_{\mu}+\partial_{\mu} \sigma$ in the Lorentz gauge

$\partial^{\alpha} \partial_{[\alpha} A_{\mu]}=c / e \partial^{\alpha} \partial_{[\alpha} u_{\mu]}=\partial^{\alpha} \partial_{\alpha} A_{\mu}$ reduced identically to the conservation law of the density spin tensor $S_{\mu \nu}^{a}$ of the self-consistent electromagnetic field $A_{\mu}$

$$
\partial_{\alpha} S_{v \mu}^{a} \equiv \partial^{\alpha}\left(u_{[v} \partial_{\alpha} u_{\mu]}\right)=0
$$


$\rho_{e}$ is an electric charge invariant density, $u_{\mu}$ is 4 -speed of particles $\left(u^{2}=1\right), e$ is an electron charge, $\partial_{\mu}$ is a partial derivative, $c$ is a speed of light in vacuum, $\sigma(x, t)$ is a gauge function.

As a result, it was found that the Lorentz busts and Euler's angles $\Theta=\Theta(\Psi), \varphi=\varphi(\Psi)$ are functions of the $\Psi$ variable which satisfies the linear wave equation: $\square \Psi=0 . \square=\partial^{\alpha} \partial_{\alpha}$ is the D'Alambert operator. An electric charge density

$$
\rho_{e}=(4 \pi e)^{-1} m c^{2}|\boldsymbol{u}|^{-2}(\boldsymbol{u} \cdot \square \boldsymbol{u}) .
$$

The current density

$$
\boldsymbol{j}=\rho_{e} \boldsymbol{u}
$$

The model is grounded on the static exact solution of Maxwell's equations. Lorentz transform one-dimensional and three-dimensional solutions appear to mean a quasistationary wave soliton type. In relation to the fulfillment of the above task, as if scattering on the Galactic plane can be used a flattened ellipsoid of rotation coordinates system: $x^{2}+y^{2}=a^{2}\left(1+\sigma^{2}\right)\left(1-\tau^{2}\right), z=a \sigma \tau, \tau \in[-1,1], \sigma \ni[0, \infty]$. Wave equation for $\Psi\left(a^{2}\left(\tau^{2}+\sigma^{2}\right)\right)^{-1} \partial_{\sigma}\left(\left(1+\sigma^{2}\right) \partial_{\sigma} \Psi\right)=0$ has a solution

$$
\Psi=4 N \operatorname{tg} \sigma,
$$

$N$ is a number of spin flips. Then an electric full invariant charge

$$
Q=4 m c^{2} a \int_{0}^{2 \pi N} \mathrm{~d} \Psi\left(\boldsymbol{u} \cdot \partial_{\Psi}^{2} \boldsymbol{u}\right)\left(e|\boldsymbol{u}|^{2}\right)^{-1} .
$$

"a" is the radius of the disk of a constant potential surface $\sigma=0$. The main problem was the selection of model Euler's angles $\Theta=\Theta(\Psi), \varphi=\varphi(\Psi)$, providing limited integral values (4) and a current. The integrals from a charge and current through a cylindrical surface are calculated on the computer.

\section{Results}

To understand the tasks in this formulation was subjected to a theoretical treatment of the pulse of a discrete source of X-ray radiation of burst in terms of currents of the ESSM, depending on the time. This is done within the frame of the space rays generation mechanism of a method of direct transformation of intergalactic gamma-rays to the proton current on spin shock-waves [14] [15] [16]. During pulse according to the theoretical model the vector of the current runs through the same range of angles in the ESSM inner configuration space and in range of Galactic longitudes. Figure 1 (see Fig. 2 in [16]) built the pulse for X-ray burst MXB 1730-335 on the artificial satellite Date of the Earth "Astron", 1983 (see links in [16]) compared with the pulse delayed from the background ( 28 counts in $0.83 \mathrm{sec}$.). A similar build to the pulse of gamma-bursts (Fig. 2 in [18]) the GBRs010412 in Figure 2 is satisfactory and improving after smoothing on the sliding window method on 5 points within $7^{\circ}$ dispersion in internal configuration space model (see Figure 2). Bursts were far from the Galactic 


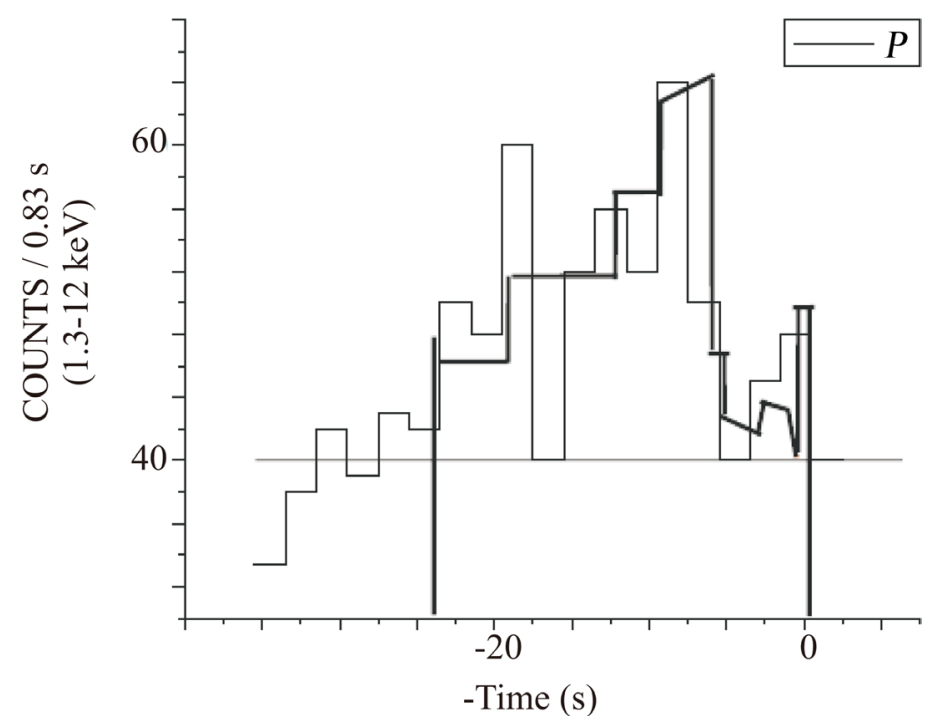

Figure 1. The X-ray burst MXB 1730-335 impulse of the first type in a map with the impulse of an Eight spin shock waves model currents [16], built from background level 40 counts for 0.83 seconds (horizontal line): theory (bold line), experiment (thin line) in the range of $1.3-12 \mathrm{keV}$.

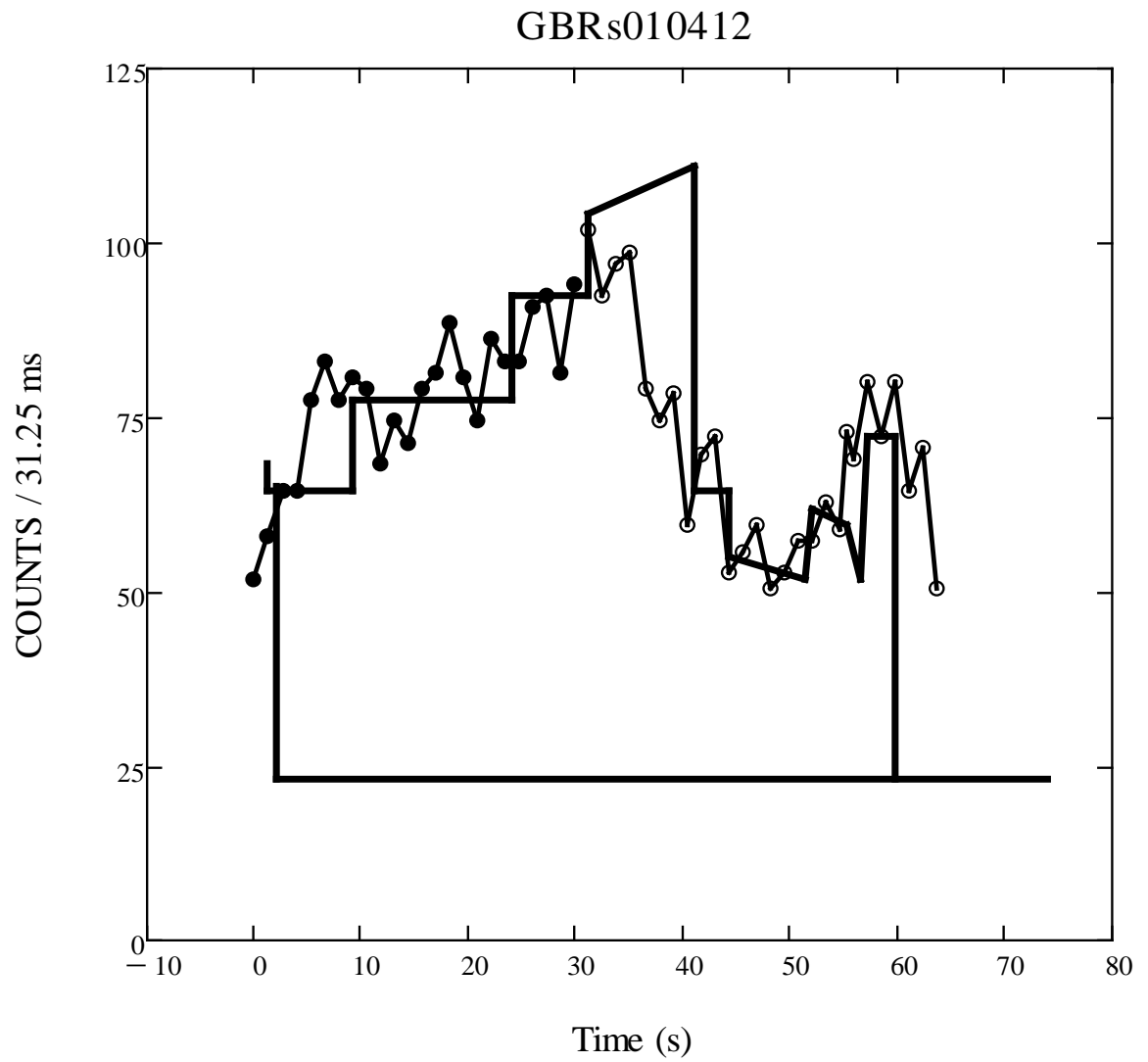

Figure 2. The gamma-ray burst GBRs010412 impulse from [18], Fig. 2, of the first type in a map with the impulse of an Eight spin shock waves model currents [15] [16] [17] [23], built from background level 23 counts for 31.825 seconds (horizontal line): theory (bold line), experiment (thin line) after smoothing on the sliding window method on 5 points (circles) and on 9 points (black circles). 
plane. Therefore, absorption in the structure of the Galaxies can be neglected. There was a precise agreement between generated currents (theory) with the burst radiation, characterized by the standard deviation of $\pm 1 \%$ in intensity in relative units within the sensitivity of the equipment. Thus, it was found that the spin angular momentum conservation law (equation of dynamics of spin shock waves) in the X-ray/gamma ranges is fulfilled exactly in real time. On the other hand gauge approach based on the connection between symmetries and conservation laws: according to the Noether's theorem conserving of spin angular momentum means the isotropy of space and time. Further, despite the fact that the source of the incident radiations is not known in advance, the axis of anisotropy can be defined, but only at the end of the match the angular distribution of the observed intensity and theory. It is shown with dates (see [14]), that the Galactic gamma-ray angular anisotropy axis has following Galactic coordinates: longitude (exactly) $l=96^{\circ}$, latitude $20^{\circ}$, that corresponds in the second equatorial coordinate system: right ascension $\alpha=271^{\circ}$, declination $\delta=40^{\circ}$. In a similar approach, according to [19] the Galactic coordinate axis of anisotropy of the angular distributions of pulsars $1=-94^{\circ}$, that upon reflection gives $1=86^{\circ}$ with $b=15^{\circ}$ in the second equatorial coordinate system: right ascension $\alpha=271^{\circ}$, declination $\delta=46^{\circ}$. There is the Supernova/GRB connection (e.g. [18]): "the GRB occurred on 25 April 1998 (GRB 980425). The event was not peculiar from the point of view of the Gamma-/X-ray phenomenology, but at its location a supernova (SN1998bw) showed up in the optical and radio band. The distance was $37 \mathrm{Mpc}(z=0.0085)$ thence the energetic was $E_{\text {iso }}=(8.5 \pm 0.1) \times 10^{47} \mathrm{erg}, 3$ or 4 orders of magnitude lower than that of typical bursts. Beside the positional coincidence, the SN explosion was (within one day) simultaneous to the GRB and, thence, the letter was likely the starting event". A similar by a catalogue supernovae [20] the coordinates of a core of supernovae are staked in required range $32^{\circ}-104^{\circ}$ and the Galactic coordinate axis of anisotropy of the angular distribution of pulsar $1=-94^{\circ}$. However, I found the surprising fact: throughout on 5000 Supernovae [20] tightly fill everywhere except for a single plot length $2^{\circ}$ in the field axis anisotropy $\alpha=271^{\circ}$. In the theory, the massive corner [21] particles defined with respect to axes of anisotropy $\mathbf{A}_{\mathrm{GS}}$ in proportion to their mass becomes zero for massless particles, photons. In this case we found that the mass (supernovae) does not appear in the $\alpha=271^{\circ}$. This and means that this place indicates the axis $\mathbf{A}_{\mathrm{GS}}$ of anisotropy of vacuum. In cases, the angular distributions anisotropy Galactic gamma-rays and pulsars using ESSM (see [21] [22] [23]) is determined by the paradoxes way, so this sets the isotropy of space-time in according to the Noether's theorem: due to the conservation of spin angular momentum, as a dynamics law for the Spin shock waves.

As know the gravitational mass defect for cusps of superconducting strings [8] is equal to $5 \mathrm{GeV} / \mathrm{c}^{2}$ on a spinor [22]. It is not enough for the prediction of the diffuse gamma-ray radiation at $10 \mathrm{GeV}-100 \mathrm{GeV}$. Thus neutron stars cannot serve as GRB engines. In another side the gravitational mass defect for supernovae systems with black holes is equal to $11 \mathrm{GeV} / \mathrm{c}^{2}$ on a spinor [22]. It is enough for the prediction of the diffuse gamma-ray radiation at $10 \mathrm{GeV}-100 \mathrm{GeV}$. For a two balls Galactic system the 
gravitational mass defect is equal to $15 \mathrm{GeV} / \mathrm{c}^{2}$ on a spinor [22]. It is enough for the prediction of the diffuse gamma-ray radiation at $10 \mathrm{GeV}-100 \mathrm{GeV}$ too.

Prospects: the origin of Fast Radio Bursts (FRBs) [24] considered that a number of Theoretical treatments of the pulses of discrete sources of X-rays/gamma-rays radiation of bursts in terms of currents of the ESSM, depending on the time, in Figure 1 and Figure 2, the next step involves setting the inverse problem of determining the wave function disturbance on the differential of measured smoothing pulses. In the asymptotic large times the problem is reduced to the solution of the functional equation with shift of the argument. This will give additional information about the change speed of the wave, as well as on interaction.

In additionally, the ultraluminous X-ray sources (ULXs) generation mechanism on a method of direct transformation of intergalactic gamma-rays (and so bursts) to the current on spin shock-waves and X-ray is FRBs can be observed as ULXs [25]. Observation of repeated fast radio bursts means that in the range of Decimetric waves has a place the same mechanism from external sources, but with different characteristics including the absorption of free electrons. Similarity X-bursts radio-bursts in theory, except for the basic types can be even 3 types. All they have to go to a certain time sequence one after another by the number $N$ of spin-flip of the electromagnetic field spin.

Analysis of formation of spin shock-waves when considering relativistic interaction in charged nonneutral plasma is given in [23]. It is shown that the mechanism of formation of the front of spin shock-waves is overturning a simple wave arising during the propagation of waves in the interelectrode gap due to the inverse proportionality of the phase velocity of a wave from its amplitude. The Eight spin shock-waves model [23], because of its local scale and Conformal invariance, in the low energy limit in the field of physics of solid turned out to be totally indistinguishable from the dual spin eight semiclassical trajectories of electrons when the magnetic breakdown of metals with spin flip. In quasy-classical approximation, the electric field potential (as well as its spin) is a classic. The fundamental nature of spin shock waves is not limited to either only from known species interaction is electromagnetic, weak, strong, or gravity, but in the general case is defined by their cumulative effects. The formation of a new kind of isospin shock waves with isospin flip from the isospin $I=+1$ (scattering goes mainly on the proton $(90 \%)$ with the isospin $I=+1)$ to $I=-1$ (scattering goes mainly on the neutron (also 90\%) with the isospin $I=-1$ ) in a deep inelastic scattering of electrons and neutrinos on the deuteron in the area of cumulative meson production demonstrated in [21] [23].

\section{Conclusions}

Thus, anyway the success of numerous predictions requires consideration of internalexternal sources. And it must still be (X-ray) afterglows. On the other hand, the nonthermal gamma-rays are supposed to be produced by a fireball of relativistic $\mathrm{e}^{-} \mathrm{e}^{+}$pairs that are created by annihilation of neutrino-antineutrino pairs in the vicinity of the hot, merged object [9]. It is also known that a large number of fermions with a pair gravita- 
tional interaction occur a spatial symmetry spontaneous breaking, accompanied by gravitational mass defect (as all know the gravitational mass defect neutron stars reached $90 \%$ ). If spherical symmetry is broken, as in the known case of the merger scenario where a rapidly rotating dick can be formed and material is pulled away from rotation axis by centrifugal forces, then a baryon-free funnel along the rotation axes may allow relativistic beams of $\gamma$ 's and $\mathrm{e}^{-} \mathrm{e}^{+}$to escape. It seems to be extremely hard to account for the energy of cosmological gamma-bursts by $v \bar{v}$ annihilations [9]. Another thing is this may occur under the influence of gamma-rays from a powerful external source. The Gamma-ray bursts origin clearly disclosed within frame of the space rays generation mechanism of a method of direct transformation of intergalactic gamma-rays to the proton current on spin shock-waves [14] [15] [16]. There is a reason to believe that the initiation of such explosive astronomical phenomenon as supernovae is the same origin, because the coordinates of a core of supernovae are staked in required range, $32^{\circ}$ $104^{\circ}$, in Galactic co-ordinates. In addition, here the surprising fact is found: 5000 supernovae [20] densely fills the space everywhere except for a single area with a length of $2^{\circ}$, situated in the area of axis of anisotropy $\alpha=271^{\circ}$. Also of interest is that cusps of superconducting strings can serve as GRBs engines [8]. As of superconducting strings in our Galaxy can serve areas mentioned spheres above, because their radii 15,000 light years coincided with the calculated radius of a superconducting condensate of gravitating fermion system on helium-3 [21].

The next step involves setting the inverse problem of determining the wave function disturbance on the differential of measured smoothing pulses. This will give additional information about the change of the speed of the wave, as well as on the interaction.

\section{References}

[1] Kawamura, T., Giacomazzo, B., Kastaun, W., Ciolfi, R., Endrizzi, A., Baiotti, L. and Perna, R. (2016) Binary Neutron Star Mergers and Short Gamma-Ray Bursts: Effects of Magnetic Field Orientation, Equation of State, and Mass Ratio. Physical Review D, 94, 064012. https://doi.org/10.1103/PhysRevD.94.064012

[2] Wiersema, K., Covino, S., Toma, K., van der Horst, A.J., et al. (2014) Circular Polarization in the Optical Afterglow of GRB 121024A. Nature, 509, 201-204. https://doi.org/10.1038/nature13237

[3] Piran, T. (2005) The Physics of Gamma-Ray Bursts. Reviews of Modern Physics, 76, 1143. https://doi.org/10.1103/RevModPhys.76.1143

[4] Dado, S. and Dar, A. (2016) Critical Test of Gamma-Ray Burst Theories. Physical Review D, 94, 063007. https://doi.org/10.1103/PhysRevD.94.063007

[5] Mitra, A. (2006) On the Non-Occurrence of Type I X-Ray Bursts from the Black Hole Candidates. Advances in Space Research, 38, 2917-2919. https://doi.org/10.1016/j.asr.2006.02.074

[6] Tewari, B.C. and Charan, K. (2015) Horizon Free Eternally Collapsing Anisotropic Radiating Star. Astrophysics and Space Science, 357, 107. https://doi.org/10.1007/s10509-015-2335-2

[7] Tewari, B.C., Charan, K. and Rami, J. (2016) Spherical Gravitational Collapse of Anisotropic Radiating Fluid Sphere. IJAA, 6, 155-165. 
https://doi.org/10.4236/ijaa.2016.62013

[8] Berezinsky, V., Hnatyk, B. and Vilenkin, A. (2001) Gamma Ray Bursts from Superconducting Cosmic Strings. Physical Review D, 64, 043004.

https://doi.org/10.1103/PhysRevD.64.043004

[9] Janka, H.-T. and Ruffert, M. (1995) Can Neutrinos from Neutron Star Mergers Power Gamma-Ray Bursts? Astronomy and Astrophysics, 307 No. 2.

[10] Syromyatnikov, A.G. (1991) Gauge Invariance Problem in Field Theory with Dynamic Torsion. Theoretical and Mathematical Physics, 87, 444-446. https://doi.org/10.1007/BF01016586

[11] Satarov, A.G. and Syromyatnikov, A.G. (1992) O nekotorih osobennostyah dvuh podhodov k affinno-metricheskoi teorii gravitatcii [On Some Features of Two Approaches to the Affine-Metric Theory of Gravitation]. Theoretical and Mathematical Physics, 92, 1, 150.

[12] Satarov, A.G. and Syromyatnikov, A.G. (1993) On Some Features of Two Approaches to the Affine-Metric Theory of Gravitation. Plenum Publishing Corporation, New York, 799-801.

[13] Norden, A.P. (1976) Spaces of the Affine Connectivity. Nauka, Moscow.

[14] Syromyatnikov, A.G. (2015) New Horizons in Nuclear Physics, Nuclear Engineering, Femto- and Nanotechnologies. Proceeding of the LXV International Conference "Nucleus-2015", Saint-Petersburg, Russia, 29 June-3 July 2015, 173.

[15] Syromyatnikov, A.G. (2015) Similarity All-Known Particle/Resonance Mass and Nuclear Atomic Weight. LAP Lambert Academic Publishing GmbH \& Co. KG, Saarbrucken, Germany.

[16] Syromyatnikov, A.G. (2012) A Possible Mechanism for the Generation of Cosmic Rays. Vestnik of Saint-Petersburg University, 4, 108-112.

[17] Syromyatnikov, A.G. (1993) Method of Self-Consistent Field in Non-Linear Dynamics Problems. Tip. VKA, St. Petersburg, Russia.

[18] Costa, E. and Frontera, F. (2011) Gamma Ray Burst Origin and Their Afterglow: Story of a Discovery and More. La Rivista del Nuovo Cimento, 34, 585-615. Arxiv:1107.1661

[19] Teilor, J.H., Manchester, R.N. and Lyne, A.G. (1993) Catalog of 558 Pulsars. The Astrophysical Journal Supplement Series, 88, 529-568.

[20] CBAT: IAU Central Bureau for Astronomical Telegrams (2005). http://www.cbat.eps.harvard.edu/lists/Supernovae.html

[21] Syromyatnikov, A.G. (2015) On Some Features of Possible Torsion Effects on Observables at Hadron Colliders. International Journal of Geometric Methods in Modern Physics, 12, 1550080.

[22] Syromyatnikov, A.G. (2016) The g-2 Muon Anomaly in Dimuon Production with the Torsion in LHC. International Journal of Geometric Methods in Modern Physics, 13, 1650093.

[23] Syromyatnikov, A.G. (2012) Physical Effects of the Conformal Gauge Theory of Gravitation. LAP Lambert Academic Publishing GmbH \& Co. KG, Saarbrucken, Germany.

[24] Spitler, L.G., Scholz, P., Hessels, J.W.T., et al. (2016) A Repeating Fast Radio Burst. Nature 531, 202-205. https://doi.org/10.1038/nature17168

[25] Popov, S.B. and Pshirkov, M.S. (2016) Fast Radio Bursts Counterparts in Scenario of Supergiant Pulses. Arxiv:1605.01992[astro-ph.HE] 
Submit or recommend next manuscript to SCIRP and we will provide best service for you:

Accepting pre-submission inquiries through Email, Facebook, LinkedIn, Twitter, etc. A wide selection of journals (inclusive of 9 subjects, more than 200 journals)

Providing 24-hour high-quality service

User-friendly online submission system

Fair and swift peer-review system

Efficient typesetting and proofreading procedure

Display of the result of downloads and visits, as well as the number of cited articles

Maximum dissemination of your research work

Submit your manuscript at: http://papersubmission.scirp.org/

Or contact ijaa@scirp.org 